Supporting Information

\title{
Theoretical studies on the Mechanism of Thioesterase-catalyzed Macrocyclization in Erythromycin Biosynthesis
}

\author{
Xiong-Ping Chen, ${ }^{1, \#}$ Ting Shi, ${ }^{1,}$ Xiao-Lei Wang, ${ }^{1}$ Qihua Chen, ${ }^{1}$ Lingquan Bai, ${ }^{1}$ \\ Yi-Lei Zhao ${ }^{1, *}$ \\ ${ }^{I}$ State Key Laboratory of Microbial Metabolism, Joint International Research Laboratory of \\ Metabolic and Developmental Sciences, School of Life Sciences and Biotechnology, Shanghai \\ Jiao Tong University, Shanghai 200240, China
}

*To whom correspondence should be addressed:

\section{Prof. Yi-Lei Zhao}

School of Life Sciences and Biotechnology

Shanghai Jiao Tong University

800 Dongchuan Road, Shanghai 200240, China

Tel/Fax: +86-21-34207190;

Email: yileizhao@sjtu.edu.cn 


\section{Contents}

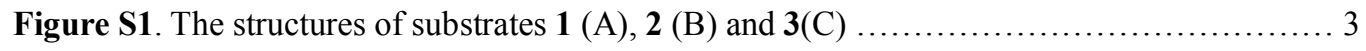

Figure S2. Comparison of initial substrate structures with (green) and without (yellow) rotation of

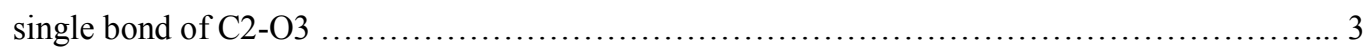

Figure S3. The water around the catalytic triad in the representative conformations of

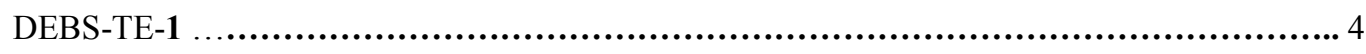

Figure S4. RMSD of 500-ns simulations for DEBS-TE-1, DEBS-TE-2 and DEBS-TE-3 …..... 4

Figure S5. Hydrogen bonding interactions and hydrophobic interactions in close $\left(\mathbf{2}_{\mathrm{I}}\right)$ and open

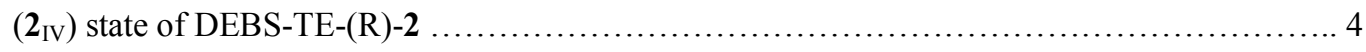

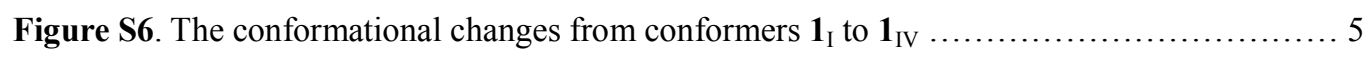

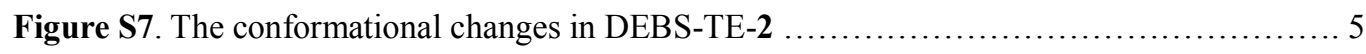

Figure S8. The conformational changes in DEBS-TE-(S)-2 show the substrate's transition from

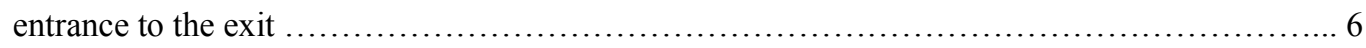

Figure S9. (A) Four representative conformations of DEBS-TE-1 in 100ns simulation. (B) The distance $\mathrm{r}_{\mathrm{O}-\mathrm{N}}$ between $\mathrm{C} 11$ hydroxyl and $\mathrm{N} \varepsilon$ of His259. (C) $\mathbf{1}_{\mathrm{IV}}$ conformation of DEBS-TE-1 . ... 7

Figure S10. The comparison of pre-reaction states in DEBS-TE-1 and DEBS-TE-3 ........... 7

Figure S11. The key structures of DEBS-TE-1 and DEBS-TE-2 along the reaction pathways .... 8

Table S1. The atom type and charge of substrate 1 ....................................... 9

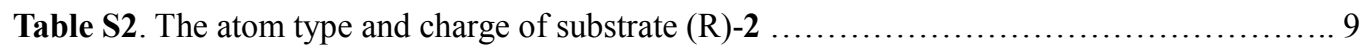

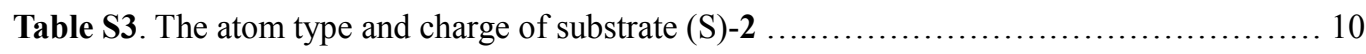

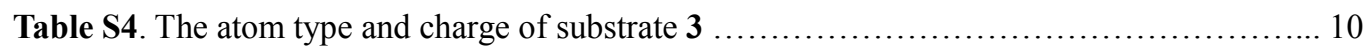

Table S5. The results obtained from 100-ns simulation of GXG (where G is glycine and $\mathrm{X}$ is substrates 1 or 2). The distance $\mathrm{r}_{\mathrm{O}-\mathrm{C}}$ is defined as the $\mathrm{C} 1$ carbonyl to the $\mathrm{C} 11$ hydroxyl. ........... 11 


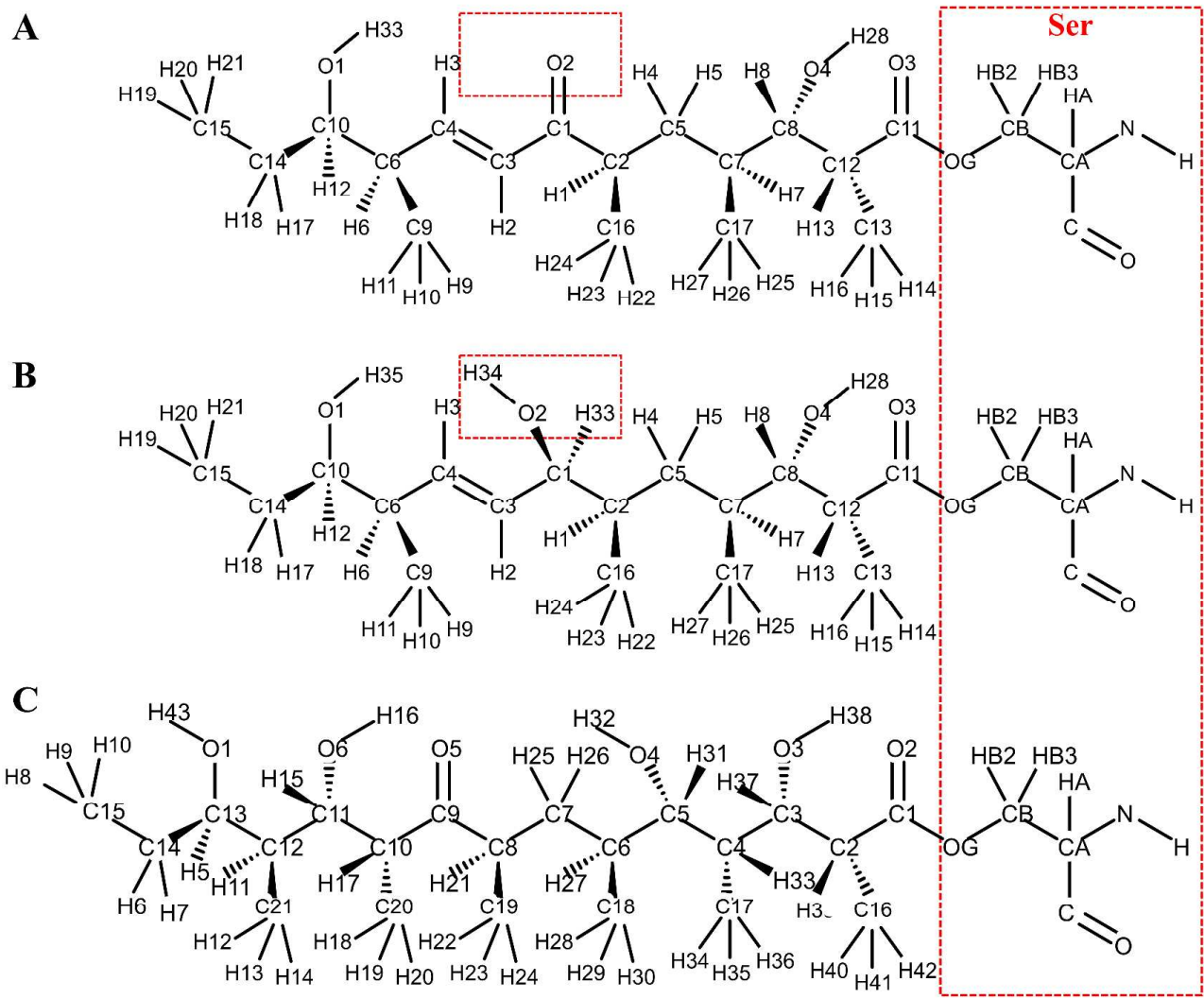

Figure S1. The structures of substrates 1 (A), 2 (B) and 3(C).
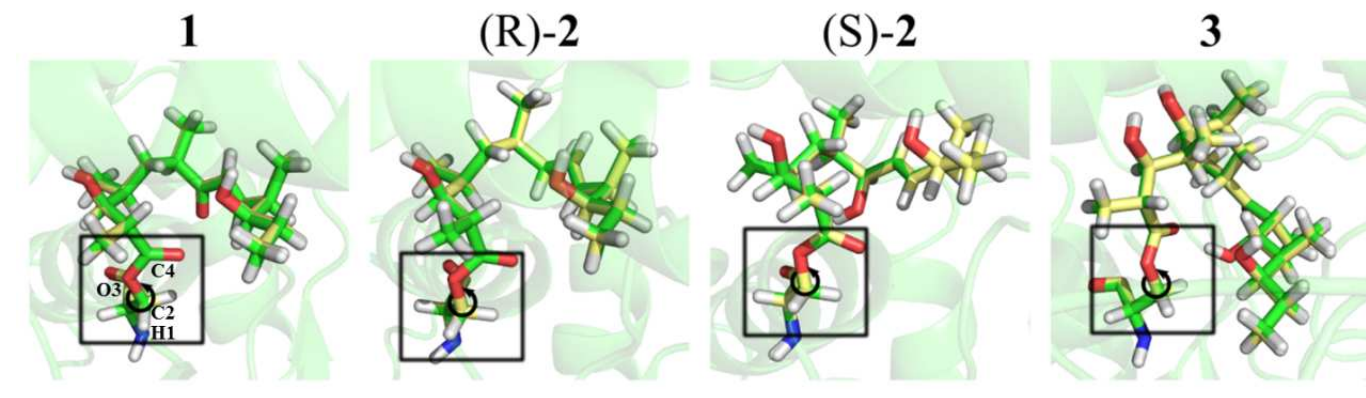

Figure S2: Comparison of initial substrate structures with (green) and without (yellow) rotation of single bond of $\mathrm{C} 2-\mathrm{O} 3$. 

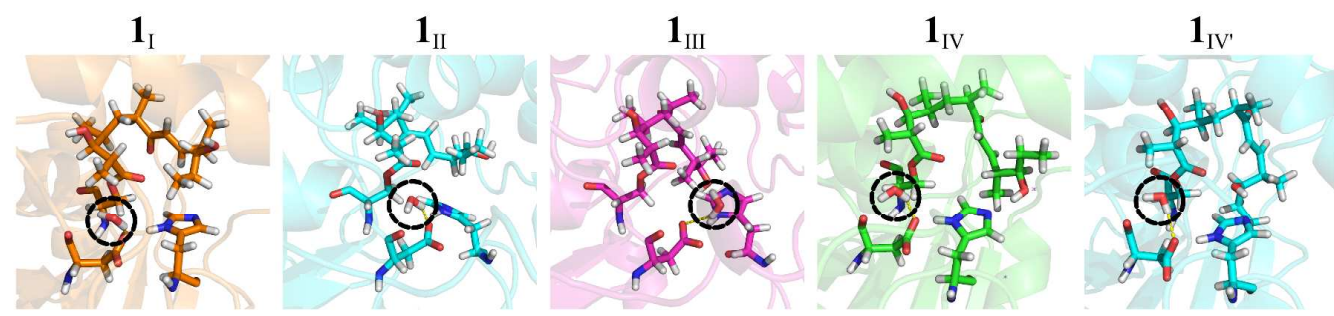

Figure S3: The water around the catalytic triad in the representative conformations of DEBS-TE-1 (1 $\mathbf{1}_{\mathrm{I}}, \mathbf{1}_{\mathrm{II}}, \mathbf{1}_{\mathrm{III}} \mathbf{1}_{\mathrm{IV}}$ and $\left.\mathbf{1}_{\mathrm{IV}}\right)$ is shown in black dash line and the hydrogen bond is shown in yellow dash line.
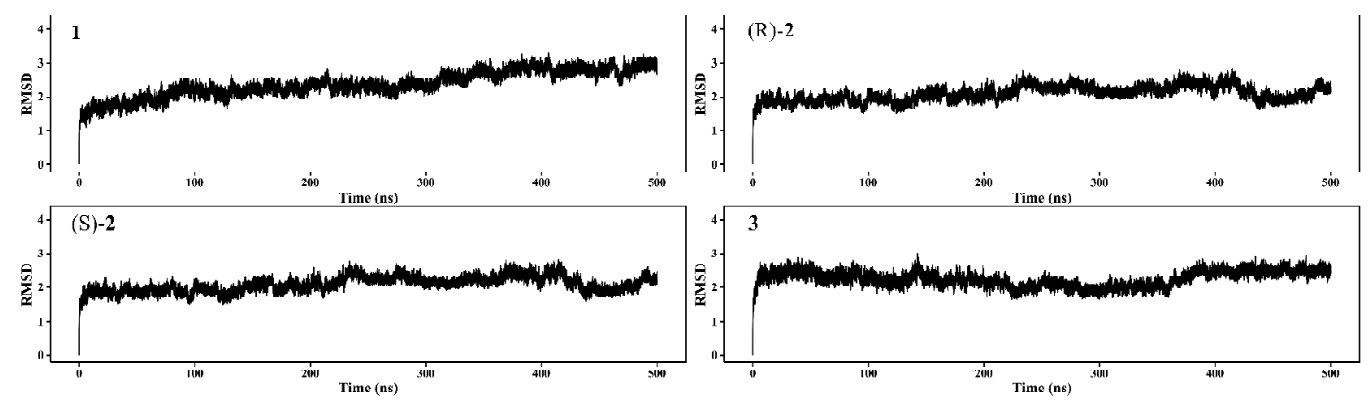

Figure S4: The RMSD of 500 ns simulations for DEBS-TE-1, DEBS-TE-2 and DEBS-TE-3. The simulations reach equilibrium after $10 \mathrm{~ns}$. To ensure the accuracy of our results, the first $20 \mathrm{~ns}$ simulation is not considered in our analysis.

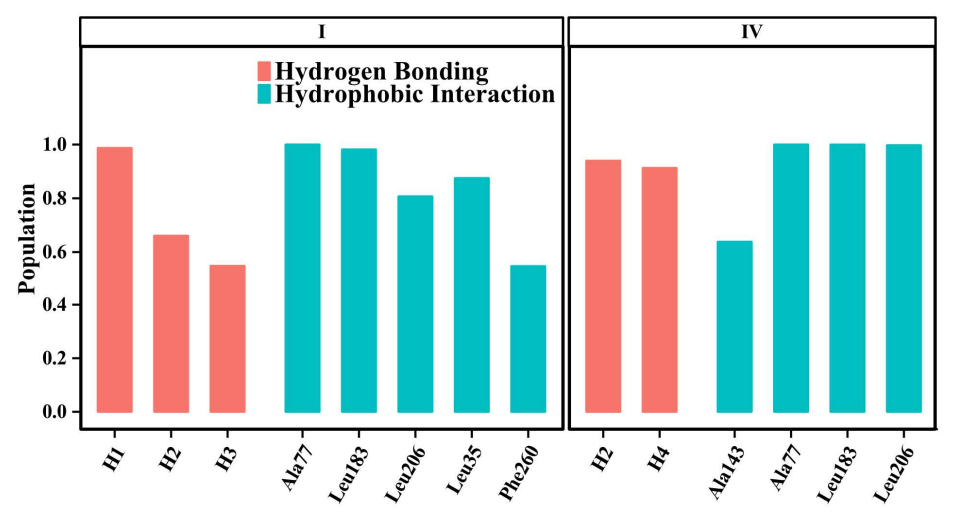

Figure S5. Hydrogen bonding interactions and hydrophobic interactions in close $\left(\mathbf{1}_{\mathrm{I}}\right)$ and open $\left(\mathbf{1}_{\mathrm{IV}}\right)$ state of DEBS-TE-(R)-2, where H1 = GLU184@OE1:(R)-2@H35; H2 = TYR171@O:(R)-2@H28; H3=(R)-2@O2--THR76@HG1; H4=ASN180@OD1:(R)-2@H35. 

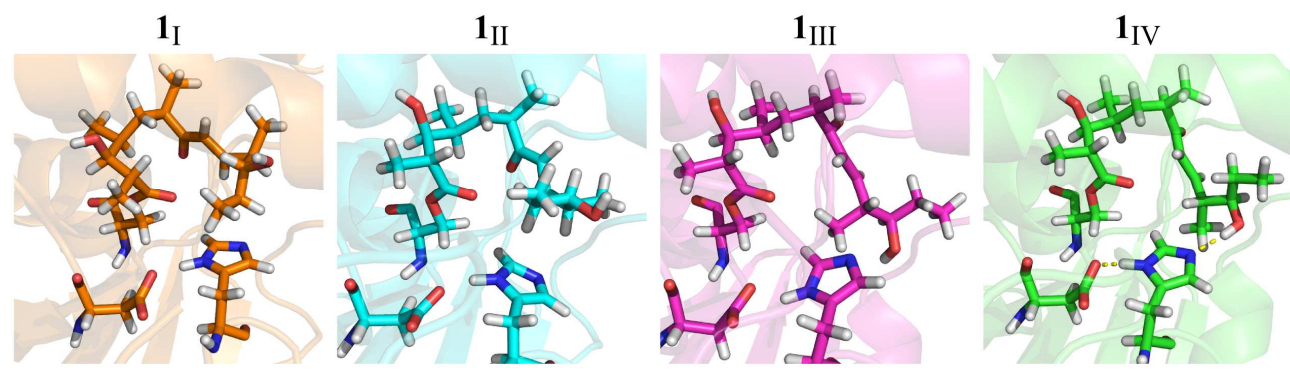

Figure S6: The conformational changes from conformer $\mathbf{1}_{\mathrm{I}}$ to $\mathbf{1}_{\mathrm{IV}}$.

A

(R)-2

(R)-2 $\mathbf{I I I}_{\mathrm{II}}$

(R)-2

(R)-2 IV

(R)-2 IV $^{\prime}$

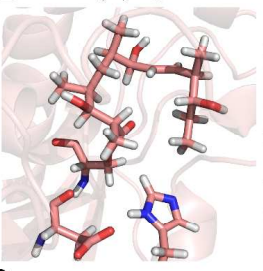

B

(S)-2

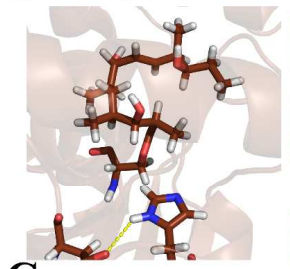

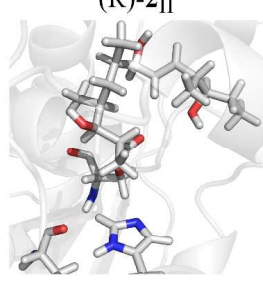

(S)-2 $\mathbf{I I}_{\mathrm{II}}$

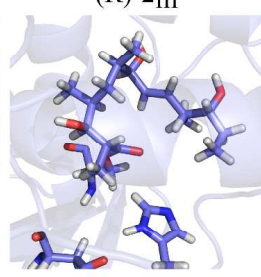

(S)-2

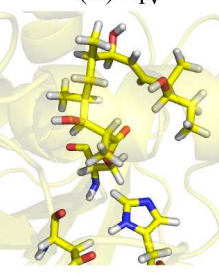

(S)-2 IV

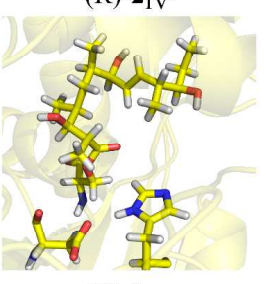

(S)-2 IV'
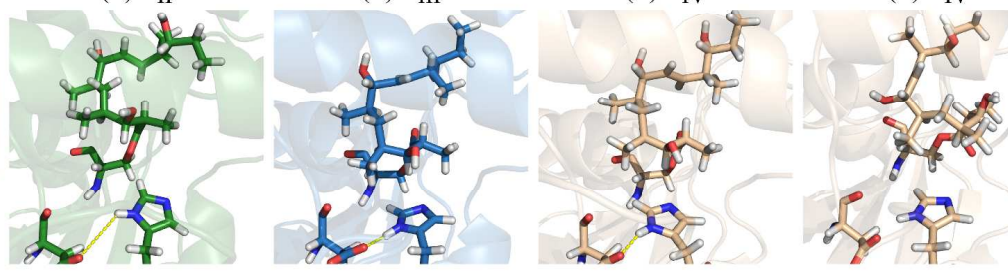

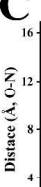

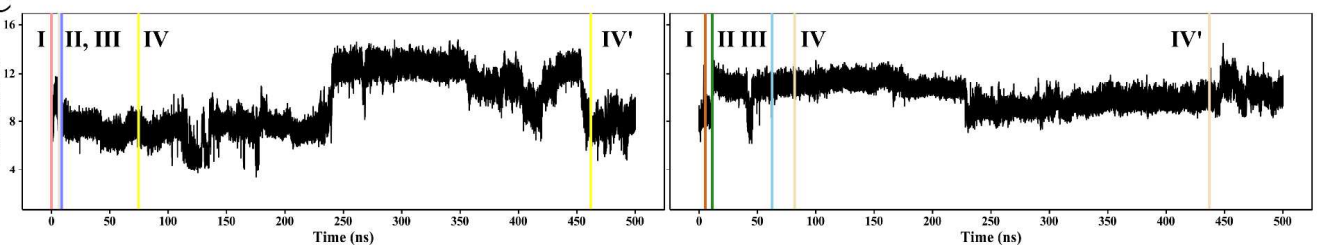

Figure S7. The conformational changes in the DEBS-TE-2. (A) The conformational changes of DEBS-TE-(R)-2 in simulation. All the structures are extracted from the representative conformations in each cluster. (B) The conformational changes of DEBS-TE-(S)-2 in simulation. (C) The distance $\mathrm{r}_{\mathrm{O}-\mathrm{N}}$ between $\mathrm{C} 11$ hydroxyl and $\mathrm{N} \varepsilon$ of His259 in DEBS-TE-(R)-2. (D) The distance $\mathrm{r}_{\mathrm{O}-\mathrm{N}}$ between $\mathrm{C} 11$ hydroxyl and $\mathrm{N} \varepsilon$ of His259 in DEBS-TE-(S)-2. 

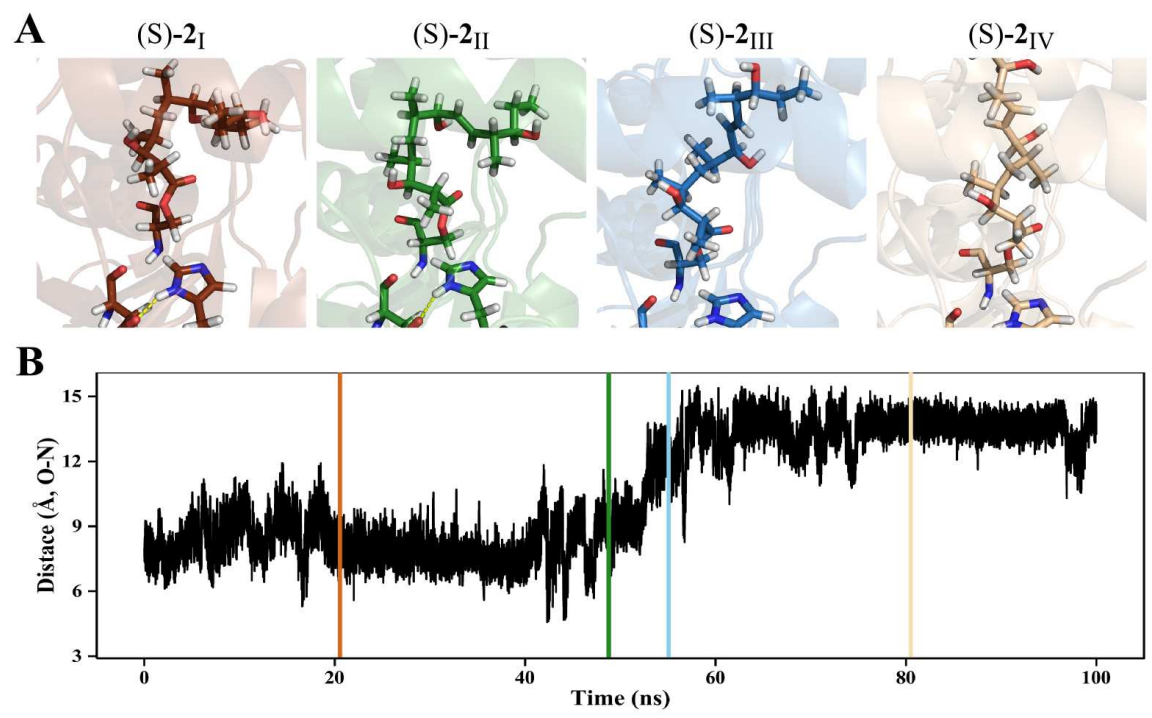

Figure S8. The conformational changes in DEBS-TE-(S)-2 show the substrate's transition from entrance to the exit.

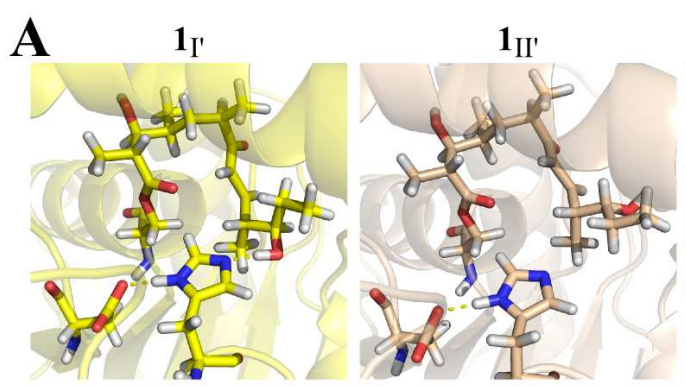

B

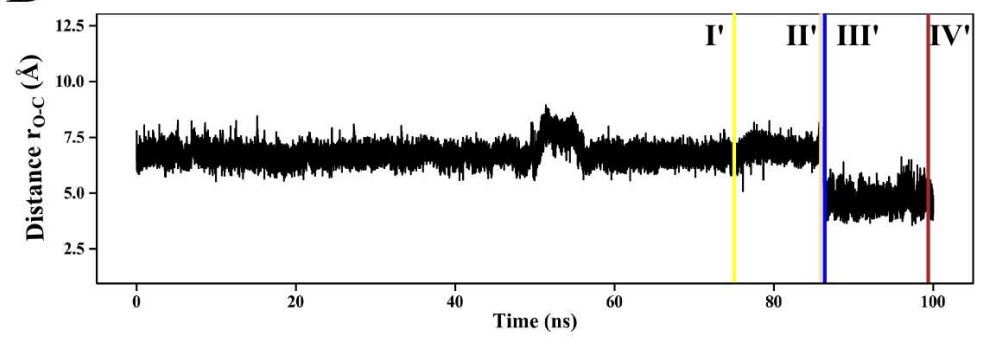

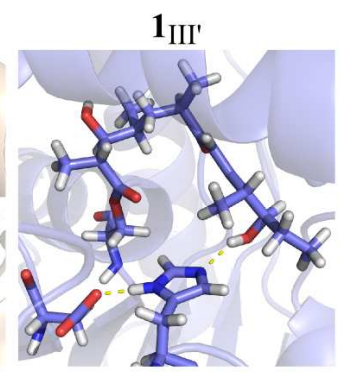

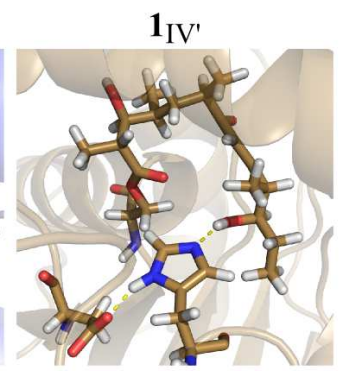

C

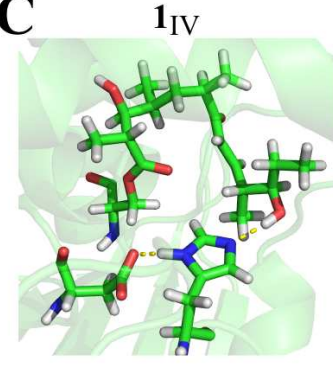

Figure S9. (A) Four representative conformations of DEBS-TE-1 in 100ns simulation. (B) The distance $\mathrm{r}_{\mathrm{O}-\mathrm{N}}$ between $\mathrm{C} 11$ hydroxyl and $\mathrm{N} \varepsilon$ of His259. (C) $\mathbf{1}_{\mathrm{IV}}$ conformation of DEBS-TE-1. 

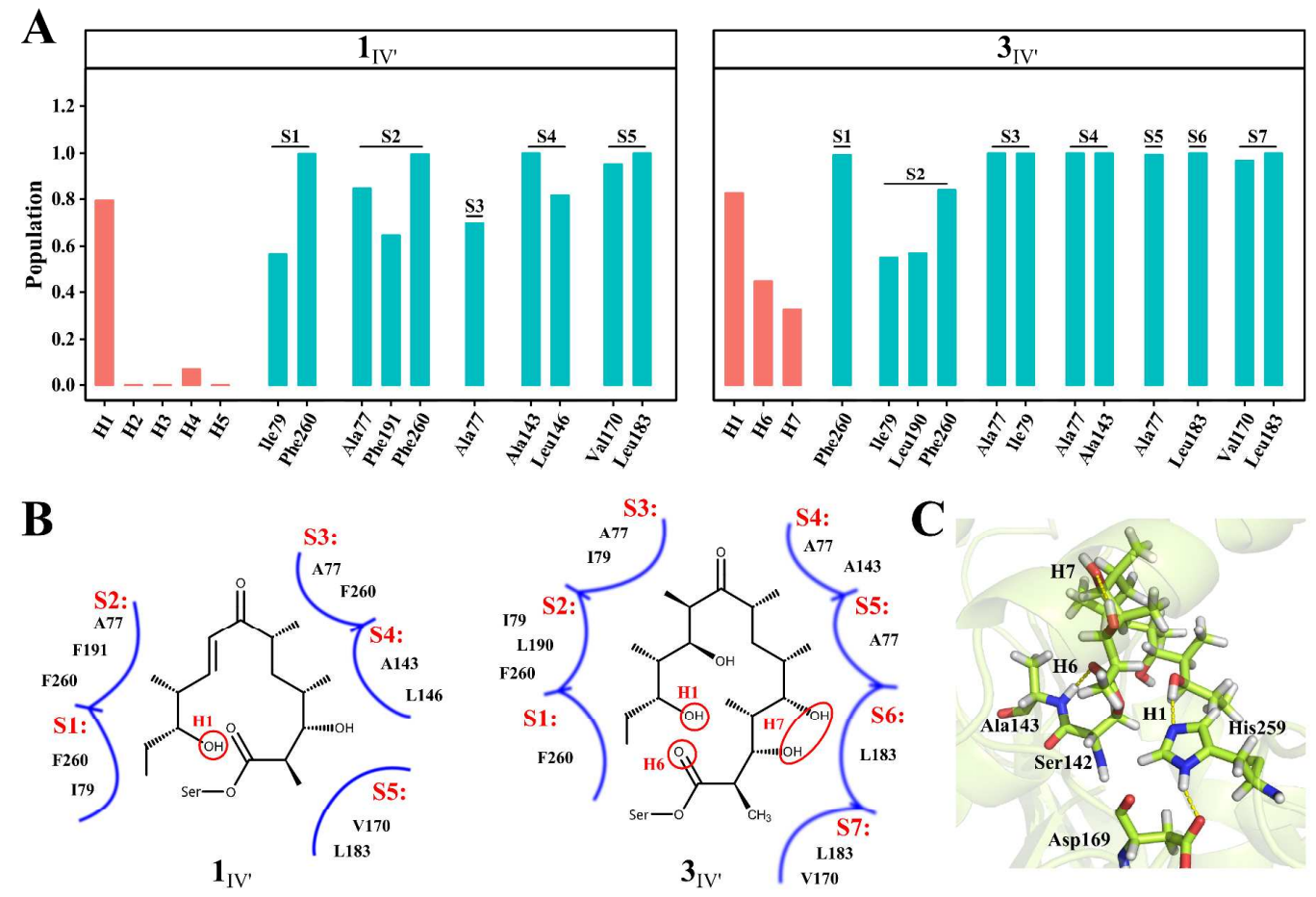

Figure S10. The comparison of pre-reaction states in substrates 1 and 3. (A) Hydrogen bonding and hydrophobic interactions in pre-reaction state. The hydrophobic interaction was classified by using the hydrophobic methyl or ethyl (S1 to S5 in substrate $\mathbf{1}$ and S1 to S7 in substrate 3). H1, the hydrogen bonding between His259 and the C11 hydroxyl in $\mathbf{1}$ or C13 hydroxyl in $\mathbf{3}$ which is the great characteristic in pre-reaction. H2=1@H33-Leu183@O,H3=1@H28-Tyr171@O, H4 = Asn180@HD22-1@O4, H5 =Arg193@HH22-1@O1, H6=Ala143@H-3@O2 (oxyanion hole), H7=3@H38-3@O4. (B) The hydrophobic and hydrogen bonding interactions of substrate 1 and 3. (C) The detailed hydrogen bonding of native substrate 3 in DEBS-TE. 


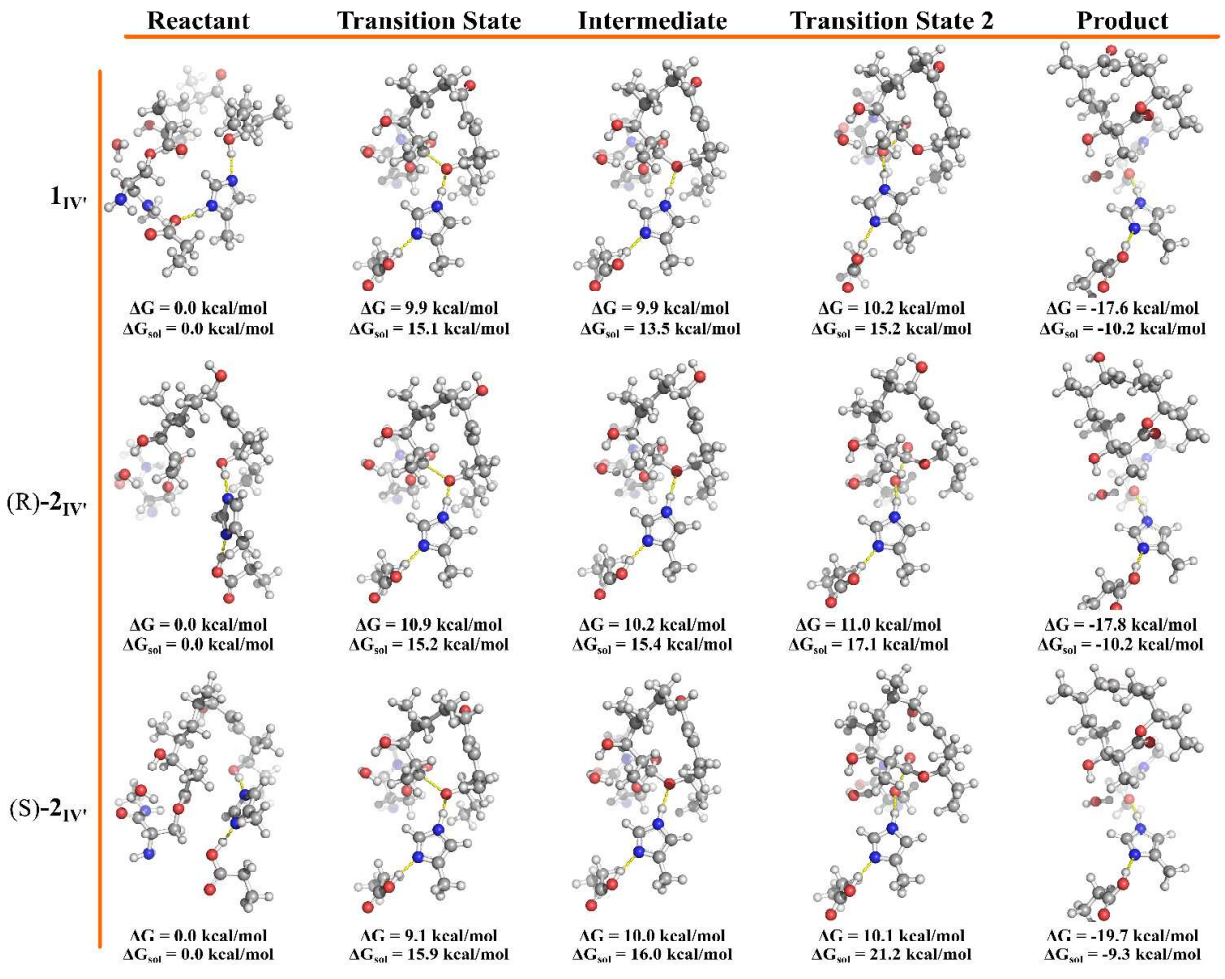

Figure S11. The key structures of substrates $\mathbf{1}$ and $\mathbf{2}$ along the reaction pathways. 
Table S1: The atom type and charge of substrate 1

\begin{tabular}{ccccccccc}
\hline Name & Type & Charge & Name & Type & Charge & Name & Type & Charge \\
\hline N & N & -0.403435 & H33 & HO & 0.413420 & H25 & HC & 0.126262 \\
H & H & 0.272924 & C14 & CT & -0.081330 & H26 & HC & 0.126262 \\
CA & CT & -0.025397 & C15 & CT & -0.158068 & H27 & HC & 0.126262 \\
CB & CT & -0.223951 & H19 & HC & 0.049608 & H7 & HC & -0.115109 \\
OG & OS & -0.346485 & H20 & HC & 0.049608 & O4 & OH & -0.730679 \\
C11 & C & 0.752886 & H21 & HC & 0.049608 & H28 & HO & 0.447275 \\
C12 & CT & 0.044361 & H17 & HC & 0.044177 & H8 & H1 & -0.040229 \\
C8 & CT & 0.383557 & H18 & HC & 0.044177 & C13 & CT & -0.552353 \\
C7 & CT & 0.013009 & H12 & H1 & 0.050956 & H14 & HC & 0.153027 \\
C5 & CT & -0.123477 & H6 & HC & 0.061743 & H15 & HC & 0.153027 \\
C2 & CT & 0.196993 & H3 & HA & 0.122656 & H16 & HC & 0.153027 \\
C1 & C & 0.506274 & H2 & HA & 0.155903 & H13 & HC & 0.094951 \\
C3 & CD & -0.353340 & O2 & O & -0.523370 & O3 & O & -0.462991 \\
C4 & CM & -0.032582 & C16 & CT & -0.395234 & HB2 & H1 & 0.157617 \\
C6 & CT & 0.032105 & H22 & HC & 0.095852 & HB3 & H1 & 0.157617 \\
C9 & CT & -0.300583 & H23 & HC & 0.095852 & HA & H1 & 0.152783 \\
H9 & HC & 0.081657 & H24 & HC & 0.095852 & C & C & 0.658841 \\
H10 & HC & 0.081657 & H1 & HC & 0.022957 & O & O & -0.612544 \\
H11 & HC & 0.081657 & H4 & HC & 0.045903 & & & \\
C10 & CT & 0.177595 & H5 & HC & 0.045903 & & & \\
O1 & OH & -0.627458 & C17 & CT & -0.467190 & & & \\
\hline
\end{tabular}

Table S2: The atom type and charge of substrate (R)-2

\begin{tabular}{ccccccccc}
\hline Name & Type & Charge & Name & Type & Charge & Name & Type & Charge \\
\hline N & N & -0.397211 & H35 & HO & 0.410788 & H5 & HC & 0.049867 \\
H & H & 0.260447 & C14 & CT & -0.139319 & C17 & CT & -0.380871 \\
CA & CT & 0.087299 & C15 & CT & -0.174063 & H25 & HC & 0.083316 \\
CB & CT & -0.485007 & H19 & HC & 0.056216 & H26 & HC & 0.083316 \\
OG & OS & -0.138736 & H20 & HC & 0.056216 & H27 & HC & 0.083316 \\
C11 & C & 0.304433 & H21 & HC & 0.056216 & H7 & HC & 0.009930 \\
C12 & CT & 0.094725 & H17 & HC & 0.063795 & O4 & OH & -0.720629 \\
C8 & CT & 0.310870 & H18 & HC & 0.063795 & H28 & HO & 0.439036 \\
C7 & CT & 0.132098 & H12 & H1 & 0.083803 & H8 & H1 & -0.001001 \\
C5 & CT & -0.170516 & H6 & HC & 0.064094 & C13 & CT & -0.572294 \\
C2 & CT & 0.136703 & H3 & HA & 0.104289 & H14 & HC & 0.160357 \\
C1 & CT & 0.321575 & H2 & HA & 0.166789 & H15 & HC & 0.160357 \\
C3 & CM & -0.316209 & O2 & OH & -0.726603 & H16 & HC & 0.160357 \\
C4 & CM & -0.140026 & H34 & HO & 0.436065 & H13 & HC & 0.113335 \\
C6 & CT & 0.036150 & H33 & H1 & 0.083345 & O3 & O & -0.424930 \\
C9 & CT & -0.265831 & C16 & CT & -0.394750 & HB2 & H1 & 0.236181 \\
H9 & HC & 0.067368 & H22 & HC & 0.090908 & HB3 & H1 & 0.236181 \\
H10 & HC & 0.067368 & H23 & HC & 0.090908 & HA & H1 & 0.128440 \\
H11 & HC & 0.067368 & H24 & HC & 0.090908 & C & C & 0.585754 \\
C10 & CT & 0.141178 & H1 & HC & 0.037381 & O & O & -0.578734 \\
O1 & OH & -0.535981 & H4 & HC & 0.049867 & & & \\
\hline
\end{tabular}


Table S3: The atom type and charge of substrate (S)-2

\begin{tabular}{ccccccccc}
\hline Name & Type & Charge & Name & Type & Charge & Name & Type & Charge \\
\hline N & N & -0.403459 & H42 & HO & 0.437064 & H5 & HC & 0.014742 \\
H & H & 0.247767 & C14 & CT & -0.098942 & C17 & CT & -0.380754 \\
CA & CT & 0.049553 & C15 & CT & -0.188169 & H25 & HC & 0.073391 \\
CB & CT & 0.191942 & H19 & HC & 0.057732 & H26 & HC & 0.073391 \\
OG & OS & -0.237416 & H20 & HC & 0.057732 & H27 & HC & 0.073391 \\
C11 & C & 0.18613 & H21 & HC & 0.057732 & H7 & HC & 0.003876 \\
C12 & CT & 0.204541 & H17 & HC & 0.059775 & O4 & OH & -0.717603 \\
C8 & CT & 0.216035 & H18 & HC & 0.059775 & H28 & HO & 0.438148 \\
C7 & CT & 0.226201 & H12 & H1 & 0.157182 & H8 & H1 & 0.054869 \\
C5 & CT & -0.059049 & H6 & HC & 0.074619 & C13 & CT & -0.56357 \\
C2 & CT & 0.196844 & H3 & HA & 0.203206 & H14 & HC & 0.14897 \\
C1 & CT & 0.165577 & H2 & HA & 0.125726 & H15 & HC & 0.14897 \\
C3 & CM & -0.134162 & O2 & OH & -0.669613 & H16 & HC & 0.14897 \\
C4 & CM & -0.248329 & H34 & HO & 0.418959 & H13 & HC & 0.109656 \\
C6 & CT & 0.036878 & H33 & H1 & 0.035825 & O3 & O & -0.428089 \\
C9 & CT & -0.270388 & C16 & CT & -0.512284 & HB2 & H1 & 0.02799 \\
H9 & HC & 0.071813 & H22 & HC & 0.12063 & HB3 & H1 & 0.02799 \\
H10 & HC & 0.071813 & H23 & HC & 0.12063 & HA & H1 & 0.106426 \\
H11 & HC & 0.071813 & H24 & HC & 0.12063 & C & C & 0.536707 \\
C10 & CT & -0.011779 & H1 & HC & -0.004255 & O & O & -0.563506 \\
O1 & OH & -0.554988 & H4 & HC & 0.014742 & & & \\
\hline
\end{tabular}

Table S4: The atom type and charge of substrate 3

\begin{tabular}{|c|c|c|c|c|c|c|c|c|}
\hline Name & Type & Charge & Name & Type & Charge & Name & Type & Charge \\
\hline $\mathrm{N}$ & $\mathrm{N}$ & -0.295778 & $\mathrm{H} 43$ & $\mathrm{HO}$ & 0.439503 & H30 & $\mathrm{HC}$ & 0.079181 \\
\hline $\mathrm{H}$ & $\mathrm{H}$ & 0.214991 & H5 & H1 & 0.025413 & $\mathrm{H} 27$ & $\mathrm{HC}$ & -0.027378 \\
\hline $\mathrm{CA}$ & $\mathrm{CT}$ & -0.242463 & $\mathrm{C} 21$ & $\mathrm{CT}$ & -0.345333 & $\mathrm{O} 4$ & $\mathrm{OH}$ & -0.702091 \\
\hline $\mathrm{CB}$ & $\mathrm{CT}$ & 0.392118 & H12 & $\mathrm{HC}$ & 0.073076 & H32 & $\mathrm{HO}$ & 0.433953 \\
\hline OG & OS & -0.403446 & H13 & $\mathrm{HC}$ & 0.073076 & H31 & H1 & 0.01499 \\
\hline $\mathrm{C} 1$ & $\mathrm{C}$ & 0.453296 & H14 & $\mathrm{HC}$ & 0.073076 & $\mathrm{C} 17$ & $\mathrm{CT}$ & -0.560108 \\
\hline $\mathrm{C} 2$ & $\mathrm{CT}$ & 0.339587 & H11 & $\mathrm{HC}$ & 0.073004 & H34 & $\mathrm{HC}$ & 0.136353 \\
\hline $\mathrm{C} 3$ & $\mathrm{CT}$ & 0.06683 & O6 & $\mathrm{OH}$ & -0.654284 & H35 & $\mathrm{HC}$ & 0.136353 \\
\hline $\mathrm{C} 4$ & $\mathrm{CT}$ & 0.511948 & H16 & $\mathrm{HO}$ & 0.443993 & H36 & $\mathrm{HC}$ & 0.136353 \\
\hline C5 & $\mathrm{CT}$ & 0.09317 & H15 & $\mathrm{H} 1$ & 0.328258 & H33 & $\mathrm{HC}$ & -0.41839 \\
\hline C6 & $\mathrm{CT}$ & 0.33398 & $\mathrm{C} 20$ & $\mathrm{CT}$ & -0.375565 & $\mathrm{O} 3$ & $\mathrm{OH}$ & -0.679372 \\
\hline $\mathrm{C} 7$ & $\mathrm{CT}$ & -0.399387 & H18 & $\mathrm{HC}$ & 0.096076 & H38 & $\mathrm{HO}$ & 0.44504 \\
\hline $\mathrm{C} 8$ & $\mathrm{CT}$ & 0.197647 & H19 & $\mathrm{HC}$ & 0.096076 & H37 & H1 & 0.030811 \\
\hline C9 & $\mathrm{C}$ & 0.399134 & $\mathrm{H} 20$ & $\mathrm{HC}$ & 0.096076 & $\mathrm{C} 16$ & $\mathrm{CT}$ & -0.446147 \\
\hline $\mathrm{C} 10$ & $\mathrm{CT}$ & 0.273054 & H17 & $\mathrm{HC}$ & -0.038039 & $\mathrm{H} 40$ & $\mathrm{HC}$ & 0.126178 \\
\hline $\mathrm{C} 11$ & $\mathrm{CT}$ & 0.016664 & $\mathrm{O} 5$ & $\mathrm{O}$ & -0.512241 & $\mathrm{H} 41$ & $\mathrm{HC}$ & 0.126178 \\
\hline $\mathrm{C} 12$ & $\mathrm{CT}$ & 0.014875 & C19 & $\mathrm{CT}$ & -0.318093 & H42 & $\mathrm{HC}$ & 0.126178 \\
\hline $\mathrm{C} 13$ & $\mathrm{CT}$ & 0.179572 & $\mathrm{H} 22$ & $\mathrm{HC}$ & 0.078134 & H39 & $\mathrm{HC}$ & 0.043519 \\
\hline $\mathrm{C} 14$ & $\mathrm{CT}$ & -0.041666 & $\mathrm{H} 23$ & $\mathrm{HC}$ & 0.078134 & $\mathrm{O} 2$ & $\mathrm{O}$ & -0.542515 \\
\hline $\mathrm{C} 15$ & $\mathrm{CT}$ & -0.235966 & $\mathrm{H} 24$ & $\mathrm{HC}$ & 0.078134 & HB2 & $\mathrm{H} 1$ & 0.013777 \\
\hline H8 & $\mathrm{HC}$ & 0.069029 & $\mathrm{H} 21$ & $\mathrm{HC}$ & 0.030346 & HB3 & $\mathrm{H} 1$ & 0.013777 \\
\hline H9 & $\mathrm{HC}$ & 0.069029 & $\mathrm{H} 25$ & $\mathrm{HC}$ & 0.075199 & HA & $\mathrm{H} 1$ & 0.167728 \\
\hline $\mathrm{H} 10$ & $\mathrm{HC}$ & 0.069029 & $\mathrm{H} 26$ & $\mathrm{HC}$ & 0.075199 & $\mathrm{C}$ & $\mathrm{C}$ & 0.579508 \\
\hline H6 & $\mathrm{HC}$ & 0.043211 & $\mathrm{C} 18$ & $\mathrm{CT}$ & -0.342506 & $\mathrm{O}$ & $\mathrm{O}$ & -0.53495 \\
\hline $\mathrm{H} 7$ & $\mathrm{HC}$ & 0.043211 & $\mathrm{H} 28$ & $\mathrm{HC}$ & 0.079181 & & & \\
\hline $\mathrm{O} 1$ & $\mathrm{OH}$ & -0.665667 & $\mathrm{H} 29$ & $\mathrm{HC}$ & 0.079181 & & & \\
\hline
\end{tabular}


Table S5: The results obtained from 100-ns simulation of GXG (where $\mathrm{G}$ is glycine and $\mathrm{X}$ is substrates 1 or 2 ). The distance $\mathrm{r}_{\mathrm{O}-\mathrm{C}}$ is defined as the $\mathrm{C} 1$ carbonyl to the $\mathrm{C} 11$ hydroxyl.

\begin{tabular}{cccc}
\hline model & Average Distance & $\begin{array}{c}\text { Populations of } \\
\text { Distance }<4\end{array}$ & $\begin{array}{c}\text { Populations of } \\
\text { Distance < 4.5 }\end{array}$ \\
\hline $\mathbf{1}$ & $7.28 \pm 0.01$ & $2.95 \%$ & $6.54 \%$ \\
$(\mathrm{R})-\mathbf{2}$ & $7.85 \pm 0.01$ & $0.78 \%$ & $1.72 \%$ \\
$(\mathrm{~S})-\mathbf{2}$ & $8.15 \pm 0.01$ & $0.79 \%$ & $4.49 \%$ \\
\hline
\end{tabular}

